\title{
Rethinking Cardiac Risk Reduction After Noncardiac Surgery: The Postoperative Carpe Diem
}

\author{
Vineet Chopra, MD, MSc ${ }^{1 *}$ and Kim A. Eagle, MD²
}

${ }^{1}$ Division of General Internal Medicine, University of Michigan Health System, Ann Arbor, Michigan; '2Division of Cardiovascular Medicine, University of Michigan Health System, Ann Arbor, Michigan.

Patients undergoing noncardiac surgery frequently experience major adverse cardiac events. As a significant proportion of these patients develop cardiac complications despite optimal use of preoperative clinical risk-prediction algorithms, physicians have long searched for better methods of forecasting and ameliorating cardiac risk in this population. Recently, postoperative troponin levels have been found to be powerful and independent predictors of cardiovascular mortality in patients undergoing noncardiac surgery. Importantly, the predictive properties of these markers outperform preoperative clinical risk-prediction algorithms. We thus posit that the assessment of postoperative troponin represents an as yet untapped "golden opportunity" for cardiac risk reduction. As cardiac troponin isolates an unusually high-risk subgroup, we outline a strategy that utilizes this marker to improve cardiac outcomes. Where pertinent, strengths and limitations of this approach are discussed and areas of uncertainty identified. As with all hypotheses, this proposition fuels many questions and calls for a research agenda dedicated to quantifying risk or benefit, and defining best practices. Journal of Hospital Medicine 2012;7:721-723. (c) 2012 Society of Hospital Medicine
Noncardiac surgery is frequently associated with major adverse cardiac events. Prevention of these events has traditionally focused on risk estimation and targeted interventions prior to surgical intervention. ${ }^{1}$ Cardiac risk is assessed through clinical prediction rules, such as the revised cardiac risk index (RCRI) or the American Society of Anesthesiology (ASA) classification. In patients deemed to be at high risk of adverse cardiac events, discretionary preoperative testing, medical treatments, and interventions are implemented. ${ }^{2}$ However, even when executed optimally, this approach fails to protect all patients. Thus, many patients undergoing noncardiac surgery continue to experience perioperative myocardial infarction (MI) and death. ${ }^{3}$

Recently, a prospective international study involving 15,133 patients reported that cardiac troponin levels measured within 3 days of noncardiac surgery were strongly associated with 30 -day mortality. ${ }^{4}$ This study is but the latest in a series of investigations that have concluded that postoperative measurement of cardiac troponin is a better predictor of cardiac outcomes than preoperative-risk algorithms. ${ }^{4-8}$ Intuitively, this link is not surprising. Surgery represents the ultimate

*Address for correspondence and reprint requests: Vineet Chopra, MD, MSc, Division of General Internal Medicine, University of Michigan Health System, 2800 Plymouth Rd, Bldg 16, Room 430W, Ann Arbor, Ml 48109-2800; Telephone: 734-647-1599; Fax: 734-936-8944; E-mail: vineetc@umich.edu

Additional Supporting Information may be found in the online version of this article.

Received: March 22, 2012; Revised: July 24, 2012; Accepted: July 25, 2012

2012 Society of Hospital Medicine DOI 10.1002/jhm.1975

Published online in Wiley Online Library (Wileyonlinelibrary.com). physiologic stress test. In the setting of hemodynamic changes and sustained oxygen supply:demand mismatch, it is hardly shocking that those with significant epicardial coronary stenosis or major subendocardial ischemia suffer poorer outcomes. What is perhaps most important about the association between troponin release and clinical outcomes, however, is the foundation it provides for a novel framework to improve perioperative care: using troponin measurement to guide treatment and interventions in the postoperative setting.

\section{MODEL TO IMPROVE POSTOPERATIVE CARDIAC OUTCOMES}

We hypothesize that the postoperative period can be seized as a "golden moment" to ameliorate cardiac risk and improve clinical outcomes for 3 reasons. First, those who release cardiac troponin during surgery declare themselves as harboring myocardial territories in jeopardy. In effect, the peripheral presence of troponin amounts to a cry for help in a population where many cardiac events are silent and thus remain clinically unnoticed. ${ }^{9,10}$ Routine postoperative monitoring for this biomarker can thus help identify a population that would ordinarily escape detection until the precipitation of a major clinical event. Second, as the pathophysiology of cardiac events centers around rupture of vulnerable plaque (type-1 MI), supply:demand mismatch (type-2 MI), or both of these phenomena, escalation or institution of important medical risk-reducing treatments (such as aspirin, beta-blockers, or statins), can be established to improve outcomes. The ability to initiate these treatments in a monitored setting may offset and address many of the risks feared with these therapies, including hypotension, stroke, or rhabdomyolysis. Third, as patients 


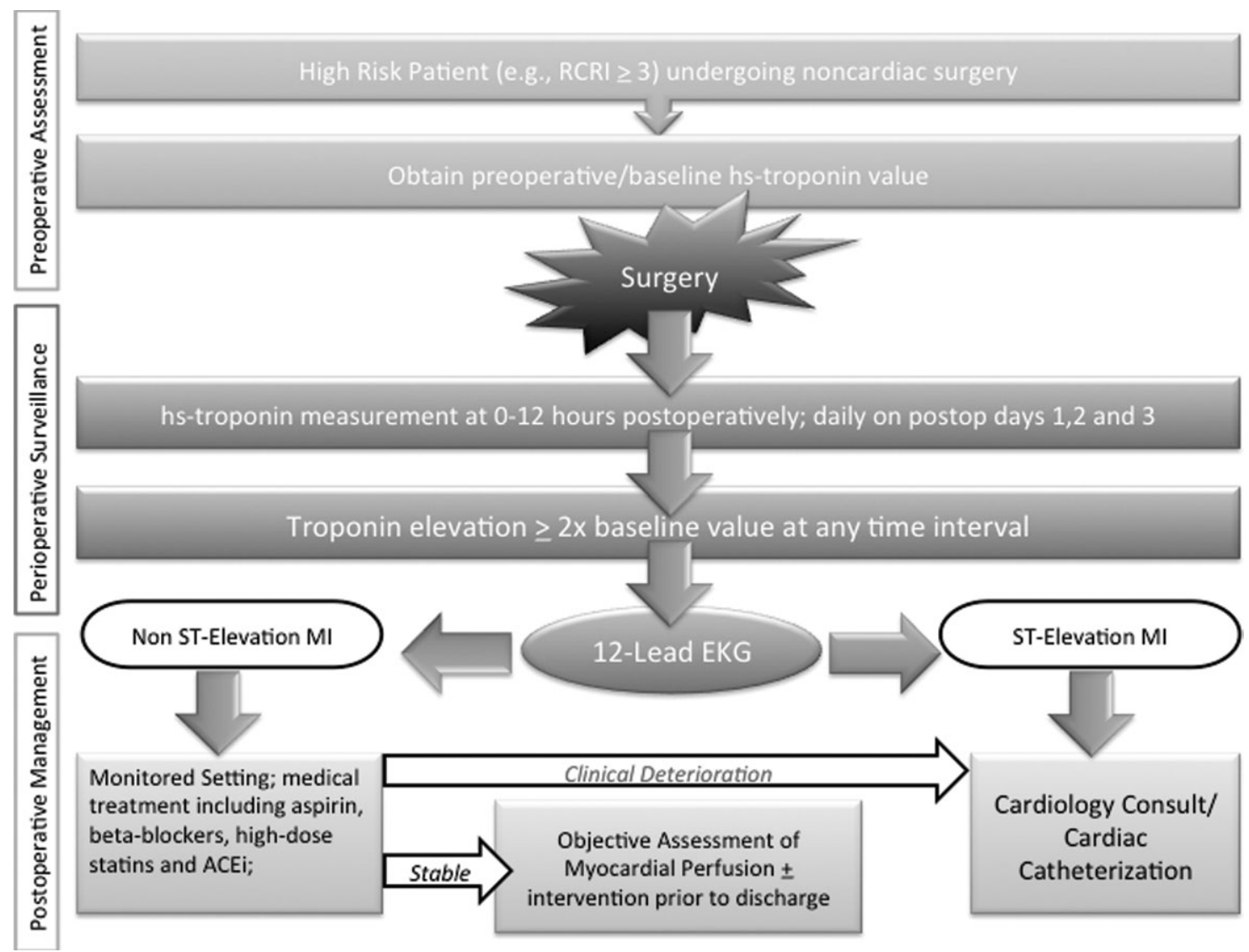

FIG. 1. Suggested pathway for implementation of cardiac troponin to improve postoperative cardiac outcomes. Abbreviations: ACEi, angiotensin-convertingenzyme inhibitors; hs-troponin; high-sensitivity troponin; MI, myocardial infarction; RCRI, revised cardiac risk index.

convalesce from surgery, timely and discretionary cardiac testing or interventions can be undertaken in those identified as being at elevated cardiovascular risk prior to discharge. In sum, the trifecta of clearly recognizing those at risk, instituting interventions in controlled settings, and performing well-timed interventions could translate into greater odds of survival in this cohort.

Owing to their intimate involvement with patients in the perioperative setting, hospitalists are uniquely suited for implementing a troponin surveillance paradigm. Yet, how may such a schema be realized? In Figure 1, we outline a pathway with which to implement this approach. This model replicates perioperative management workflow, calling for 3 discrete interventions-preoperative assessment, perioperative surveillance, and postoperative risk stratification:

1. Preoperative assessment: Patients estimated to be at high risk of perioperative cardiac events (eg, RCRI $\geq 3$ ) should undergo baseline, preoperative high-sensitivity troponin measurement, as the frequency of major adverse cardiac outcomes is significant this group $(\sim 11 \%) .{ }^{11}$ The importance of a baseline measurement of troponin cannot be overstated, as biologic variation of this marker can impact subsequent management. ${ }^{12,13}$ Hospitalists can obtain these assays in preoperative clinics at the time of initial review and risk-estimation.

2. Perioperative surveillance: Following the model implemented by a recent study, serial troponin surveillance should commence 6-12 hours after surgery, then daily on the first, second, and third day. ${ }^{4}$ As the optimal cardiac troponin threshold for the diagnosis of perioperative ischemia remains uncertain, doubling of baseline troponin levels may serve as a logical signal for further evaluation. ${ }^{14}$ The electrocardiographic pattern may be used as a branch point for immediate management at this stage: ST-segment elevation MI may be treated aggressively with an early invasive strategy and cardiac catheterization when apropos (much as in the nonoperative context). However, as most perioperative cardiac events are non-ST elevation MIs, treatment of this subset should center on initiation or escalation of medical therapy. Thus, achieving heart-rate control in order to preserve coronary perfusion by careful titration of betablockers, and initiation/up-titration of statins to temper unstable atherosclerotic plaques represent cornerstones of this approach. Other potential therapeutic entities such as initiation of angiotensin-converting-enzyme (ACE)-inhibitors in the context of left-ventricular dysfunction, and/or antiplatelet agents such as aspirin, may also be relevant risk-reducing tools.

3. Postoperative risk stratification: Prior to hospital discharge, objective assessment of myocardial perfusion and viability should occur in selected individuals with troponin elevations. Modalities that may be utilized in this context include either noninvasive imaging or occasional coronary angiography in patients with unstable 
coronary syndromes. Defining an optimal approach must be contextual, as interventions that may subsequently mandate uninterrupted antiplatelet treatment may be logistically challenging in this setting.

\section{LIMITATIONS}

Although this implementation model is plausible, it generates many questions that must be tested through the lens of a randomized controlled study. For instance, what is the optimal strategy for intervention in the postoperative setting? Though medical treatment with statins and beta-blockade may represent the mainstay of treatment, are these therapies safe during potential clinical instability? Can net benefit be realized through judicious use of coronary angiography and revascularization? Can the artifact of heightened awareness and reporting of postoperative cardiac events mar the reported quality of a hospital? Finally, though cardiac troponin has been shown to be a strong and independent predictor of mortality, which troponin assay, reference range, and troponometric standard to use remain unclear. ${ }^{15}$ Whether or not these interventions translate into lesser mortality and improved clinical outcomes represents the raison d'etre for this experimental approach. As no alternative approach to define and target patients at high risk of adverse outcome after seemingly "uneventful" surgery currently exists, we believe that this hypothetical paradigm is worthy of further investigation.

\section{CONCLUSION}

For decades, perioperative practitioners have searched for a divining rod to reduce risk during surgery. While our efforts have been focused on the preoperative setting, the postoperative setting represents an as yet untapped and potentially profound period in the quest to improving surgical outcomes. Through our proximity to patients in the perioperative setting, hospitalists are ideal agents to test, deliver, and bring about this change. It is time for a postoperative carpe diem.

Disclosure: Nothing to report.

\section{References}

1. Boersma E, Poldermans D, Bax JJ, et al. Predictors of cardiac events after major vascular surgery: role of clinical characteristics, dobutamine echocardiography, and beta-blocker therapy. JAMA. 2001; 285(14):1865-1873.

2. Eagle KA, Coley CM, Newell JB, et al. Combining clinical and thallium data optimizes preoperative assessment of cardiac risk before major vascular surgery. Ann Intern Med. 1989;110(11):859-866.

3. Weiser TG, Regenbogen SE, Thompson KD, et al. An estimation of the global volume of surgery: a modelling strategy based on available data. Lancet. 2008;372(9633):139-144.

4. VISION Study Investigators. Association between postoperative troponin levels and 30-day mortlality among patients undergoing noncardiac surgery. JAMA. 2012;307(21):2295-2304.

5. Kouvelos GN, Milionis HJ, Arnaoutoglou EM, et al. Postoperative levels of cardiac troponin versus CK-MB and high-sensitivity C-reactive protein for the prediction of 1-year cardiovascular outcome in patients undergoing vascular surgery. Coron Artery Dis. 2011;22(6):428-434.

6. Marston N, Brenes J, Garcia S, et al. Peak postoperative troponin levels outperform preoperative cardiac risk indices as predictors of longterm mortality after vascular surgery Troponins and postoperative outcomes. J Crit Care. 2012;27(1):66-72.

7. Levy M, Heels-Ansdell D, Hiralal R, et al. Prognostic value of troponin and creatine kinase muscle and brain isoenzyme measurement after noncardiac surgery: a systematic review and meta-analysis. Anesthesiology. 2011;114(4):796-806.

8. Redfern G, Rodseth RN, Biccard BM. Outcomes in vascular surgical patients with isolated postoperative troponin leak: a meta-analysis. Anaesthesia. 2011;66(7):604-610.

9. Devereaux PJ, Xavier D, Pogue J, et al. Characteristics and shortterm prognosis of perioperative myocardial infarction in patients undergoing noncardiac surgery: a cohort study. Ann Intern Med. 2011;154(8):523-528.

10. Alcock RF, Kouzios D, Naoum C, Hillis GS, Brieger DB. Perioperative myocardial necrosis in patients at high cardiovascular risk undergoing elective non-cardiac surgery. Heart. 2012;98(10):792-798.

11. Lee TH, Marcantonio ER, Mangione CM, et al. Derivation and prospective validation of a simple index for prediction of cardiac risk of major noncardiac surgery. Circulation. 1999;100(10):1043-1049.

12. Vasile VC, Saenger AK, Kroning JM, Klee GG, Jaffe AS. Biologic variation of a novel cardiac troponin I assay. Clin Chem. 2011;57(7):1080-1081.

13. Wu AH, Lu QA, Todd J, Moecks J, Wians F. Short- and long-term biological variation in cardiac troponin I measured with a high-sensitivity assay: implications for clinical practice. Clin Chem. 2009;55(1):52-58.

14. Howell SJ, Thompson JP, Nimmo AF, et al. Relationship between perioperative troponin elevation and other indicators of myocardial injury in vascular surgery patients. Br J Anaesth. 2006;96(3):303-309.

15. Ranasinghe AM, Quinn DW, Richardson M, et al. Which troponometric best predicts midterm outcome after coronary artery bypass graft surgery? Ann Thorac Surg. 2011;91(6):1860-1867. 